\title{
On the contribution of mixed terms in response function treatment of vibrational nonlinear optical properties
}

\author{
BERNARD KIRTMAN,${ }^{1}$ JOSEP M. LUIS ${ }^{2, \text { a }}$ \\ ${ }^{1}$ Department of Chemistry and Biochemistry, University of California, Santa Barbara, \\ California 93106. \\ ${ }^{2}$ Institute of Computational Chemistry and Department of Chemistry, University of Girona, \\ Campus de Montilivi, 17071 Girona, Catalonia, Spain.
}

Abstract: A new response theory formulation for calculating second-order vibrational nonlinear optical properties has recently been presented by Hansen, et al. J. Chem. Phys. 2009, 131, 154101. For so-called mixed terms this method contains a correction to account for an approximation made in the classic Bishop and Kirtman, J. Chem. Phys. 1991, 15, 2646 perturbation treatment. This correction vanishes in the static limit and is here shown to vanish also in the high frequency limit. The response theory formulation is extended to third-order properties and, in that case, vanishes in both limits as well.

Key words: Nonlinear optical properties, vibrational hyperpolarizabilites, response function.

\footnotetext{
a Correspondence to: josepm.luis@udg.edu
} 


\section{Introduction}

This paper is dedicated to the memory of David M. Bishop (DMB) our dear friend and scientific collaborator. It owes its provenance to DMB's long term interest in vibrational linear and nonlinear optical (NLO) properties $^{1-2}$ and, in particular, to the Bishop and Kirtman (BK) perturbation treatment of these quantities. ${ }^{3-5}$ This treatment has paved the way for a string of theoretical advances and $a b$ initio computations on polyatomic molecules. ${ }^{6-47}$ For conjugated organic molecules, and/or molecules with large amplitude nuclear motions, the vibrational contributions to nonlinear optical properties may dwarf those that have a pure electronic origin. ${ }^{48-50}$ Especially for the former type of molecule, this is of considerable interest due to potential utilization of the NLO properties in a variety of optical and opto-electronic devices. ${ }^{51-52}$

One effective computational procedure, applicable to the most important NLO processes, is the finite field-nuclear relaxation (FF-NR) approach. ${ }^{26,31,33,35}$ This approach is underpinned by the BK perturbation treatment and was originally developed by DMB and co-workers ${ }^{33}$ as an alternative method for obtaining the lowest-order non-vanishing perturbation terms (defined by a classification scheme whereby these terms are divided into different so-called "square bracket" types.). Subsequently, the FF-NR approach was generalized by DMB and co-workers so as to provide access to the entire vibrational contribution. ${ }^{31}$ It is important to note that the FF-NR procedure is based on a variational approach. ${ }^{33,35}$ However, it can be combined either with perturbation theory ${ }^{49,53}$ or variational methods ${ }^{8,24,38-39}$ to calculate vibrational NLO properties. Thus, in the latter case, it can be applied even when the BK perturbation treatment (based on a double harmonic oscillator zeroth-order model) converges slowly or is non-convergent. Although the FFNR method can be applied to calculate vibrational NLO properties at any optical frequency, ${ }^{21}$ for dynamic processes the most efficient implementation utilizes the infinite optical frequency (IOF) 
approximation. Despite the fact that the FF-NR treatment groups together terms of different order in perturbation theory, the exact same terms appear in either procedure (except for those eliminated by the IOF approximation) ${ }^{26}$ The latter approximation corresponds to assuming that the ratio $\left(\omega_{2} / \omega\right)^{2}$ is negligible compared to unity when $\omega$ is an (external) laser optical frequency and $\omega$, is a vibrational transition frequency within the ground electronic state. Where tests have been carried out, it has been found that this approximation is accurate as long as the optical frequencies are well above the infrared region. ${ }^{6,19,54}$

Recently, the response function formalism for molecular properties has been extended to include vibrational, along with electronic, NLO properties. ${ }^{8,24,55}$ This provides another alternative computational procedure that, like the FF-NR approach, is underpinned by BK perturbation. Nevertheless, unlike the BK perturbation treatment (and FF-NR), it does not assume that electronic transition frequencies are much larger than laser optical frequencies when the latter are in the nonresonant regime. Otherwise, however, the response formulation is entirely equivalent to these earlier approaches even though it is organized quite differently. As a result of the comparison between response and BK perturbation formulas a particular contribution has been identified as missing from the original BK perturbation method (and FF-NR approach). ${ }^{8}$ The missing contribution is due solely to the approximation mentioned just above. This approximation causes the sums over electronic states in the so-called mixed terms to be evaluated in the static limit and thereby circumvents significant computational difficulties associated with determining their frequency-dependence. This computational handicap is partially accounted for by the response treatment. At any rate, the missing contribution clearly vanishes in the static limit. A question of interest, then, is the magnitude of the missing term specifically identified by Hansen, et al. in the opposite limit, i.e. in the limit determined by the IOF approximation. The answer to this question is the subject of the 
current paper.

In the next section we present a brief review of the response formulation for the dynamic vibrational first hyperpolarizability. This includes a definition of the mixed terms and of the contribution identified in Ref. ${ }^{8}$ as missing from the BK perturbation treatment. Then, the work of Hansen et $a .^{8}{ }^{8}$ is extended in Sec. 3 to the vibrational second hyperpolarizability. In Sec. 4 we show that the missing BK terms vanish completely within the IOF approximation for both the first and second hyperpolarizability. Finally, we present our conclusions and perspective in Sec. 5.

\section{Brief review of response formulation for dynamic vibrational polarizability and first hyperpolarizability.}

We begin with the BK formulas for dynamic polarizability and first hyperpolarizability expressed as a sum over vibronic Born-Oppenheimer states. In the response theory notation of Hansen, et al. ${ }^{8}$ these are given by:

$$
\begin{gathered}
\langle\langle, Y\rangle\rangle_{\omega_{y}}=P^{X Y} \sum_{\left(I, i_{I}\right) \neq 0_{0}} \frac{\left.\left.0_{0}\left|\langle|X| I\rangle i_{I}\right\rangle i_{I}|| I|Y| 0\right\rangle 0_{0}\right\rangle}{\omega_{y}-\omega_{i_{I}}} \\
\langle X, Y, Z\rangle\rangle_{\omega_{y}, \omega_{z}}=P^{X Y Z} \sum_{\left(I, i_{I}\right),\left(J, j_{J}\right) \neq 0_{0}} \frac{\left.\left.\left.0_{0}|0| X|I\rangle i_{I}\right\rangle i_{I}|I| Y|J\rangle j_{J}\right\rangle j_{J}|J| Z|0\rangle 0_{0}\right\rangle}{\left(\omega_{i_{I}}+\omega_{x}\right)\left(\omega_{j_{J}}-\omega_{z}\right)}
\end{gathered}
$$

Here $X, Y, Z$. are the dipole moment operators $\hat{\mu}_{x}, \hat{\mu}_{y}, \hat{\mu}_{z}$ with associated frequencies $\omega_{x}, \omega_{y}, \omega_{z}$. Thus, the linear and quadratic response functions of Eqs. (1) and (2) are, respectively, the dynamic (frequency-dependent) linear polarizability and first hyperpolarizability. On the right hand side $\left.|I\rangle i_{I}\right\rangle$ is a vibronic product wavefunction in which $|I\rangle$ is the pure electronic component and $\left|i_{I}\right\rangle$ is

the ith vibrational wavefunction for electronic state $|I\rangle ; P^{X Y Z}$ represents, for example, a sum over 
the six permutations of the pairs $\left(\omega_{x} / X\right),\left(\omega_{y} / Y\right),\left(\omega_{z} / Z\right)$; and $\bar{Y}$ is the fluctuation potential $\left.Y-0_{0}|0| Y|0\rangle 0_{0}\right\rangle$. The vibrational contributions arise from those terms in the sum over states that correspond to vibrational excitations in the ground electronic state, i.e. in $|I\rangle=0$. One set of vibrational contributions corresponds to the case where there are no electronic excitations. All remaining vibrational contributions, where one or more of the electronic indices refers to an excited state, are considered to be mixed terms.

In the BK treatment (and in the response formulation) it is assumed that vibrational energy differences are small compared to electronic energy differences and, therefore, that $\omega_{i_{I}}=\omega_{I}$ (I.NE.0). This makes it possible to carry out a closure over vibrational states in the mixed for I.NE.0 to obtain the square bracket contribution:

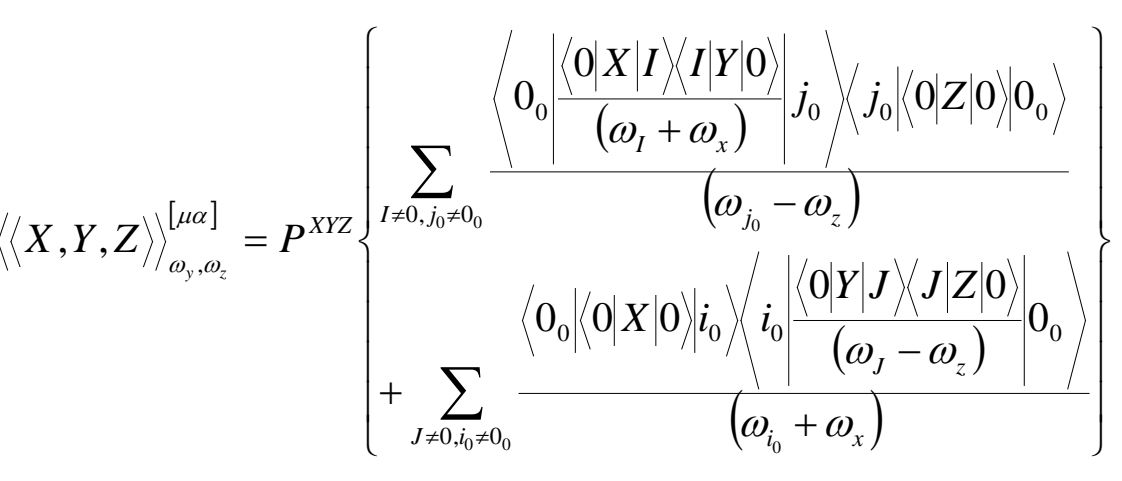

from Eq. (2).

In the response formalism of Hansen, et al. ${ }^{8}$ the square bracket in Eq. (3) is re-written in terms of the symmetric and antisymmetric quadratic electronic response functions defined, respectively, by:

$$
\langle Y, Z\rangle_{\omega}=\sum_{J \neq 0}\left\{\frac{0|Y| J\rangle J|Z| 0\rangle}{\left(\omega-\omega_{J}\right)}+\frac{0|Z| J\rangle J|Y| 0\rangle}{\left(-\omega-\omega_{J}\right)}\right\}
$$


and

$$
\langle Y, Z\rangle_{\omega}^{-}=\sum_{J \neq 0}\left\{\frac{\langle|Y| J\rangle J|Z| 0\rangle}{\left(\omega-\omega_{J}\right)}-\frac{0|Z| J\rangle J|Y| 0\rangle}{\left(-\omega-\omega_{J}\right)}\right\}
$$

From these definitions it may be readily verified that

$$
\begin{aligned}
& \left.\sum_{J \neq 0} \frac{0|Y| J\rangle J|Z| 0\rangle}{\left(\omega-\omega_{J}\right)}=\frac{1}{2}\left\{\langle, Z\rangle_{\omega}+\langle Y, Z\rangle\right\rangle_{\omega}^{-}\right\} \\
& \sum_{J \neq 0} \frac{0|Y| J\rangle J|Z| 0\rangle}{\left(-\omega-\omega_{J}\right)}=\frac{1}{2}\left\{\langle Z, Y\rangle_{\omega}-\langle Z, Y\rangle_{\omega}^{-}\right\}
\end{aligned}
$$

and, further, that

$$
\begin{aligned}
& \left\langle\langle Y, Z\rangle_{\omega}=\langle\langle, Y\rangle\rangle_{-\omega}\right. \\
& \left.\langle Y, Z\rangle\rangle_{\omega}^{-}=-\langle Z, Y\rangle\right\rangle_{-\omega}^{-}
\end{aligned}
$$

and

$$
\langle Y, Z\rangle_{0}^{-}=0
$$

Equations (4) - (10) are valid either for sums over electronic states (superindex “e” below) or for sums over the vibrational levels of the ground electronic state (superindex "v" below). In terms of the above definitions Hansen, et al. ${ }^{8}$ find that the square bracket term in Eq. (3) can be expressed as

$$
\left.\left.\left.\left.\langle X, Y, Z\rangle\rangle_{\omega_{y}, \omega_{z}}^{[\mu \alpha]}=\frac{1}{2} P^{X Y Z}\left\{\left\langle\langle X\rangle^{e},\langle Y, Z\rangle\right\rangle_{\omega_{z}}^{e}\right\rangle\right\rangle_{-\omega_{x}}^{v}+\left\langle\langle X\rangle^{e},\langle Y, Z\rangle\right\rangle_{\omega_{z}}^{e-}\right\rangle\right\rangle_{-\omega_{x}}^{v-}\right\}
$$

It follows from Eq. (10) that the second term on the rhs of Eq. (11) vanishes when $\omega_{z}=0$. This is the term identified in Ref. ${ }^{8}$ as the missing contribution in the original BK formulation. In Sec. IV we will show that this term also vanishes in the high frequency limit when the IOF approximation is 
applied.

The missing term in Eq. (11) is the only one that was considered by Hansen, et al. ${ }^{8}$ In the next section we will identify the missing terms for the dynamic vibrational second hyperpolarizability.

\section{Extension to vibrational second hyperpolarizability}

In the response theory notation the dynamic second hyperpolarizability is given by:

$$
\begin{aligned}
& \langle\langle X, Y, Z, U\rangle\rangle_{\omega_{y}, \omega_{z}, \omega_{u}}=P^{X Y Z U} \\
& \times\left\{\sum_{\left(I, i_{I}\right),\left(J, j_{J}\right),\left(K, k_{K}\right) \neq 0_{0}} \frac{\left\langle 0_{0} \mid\langle 0|X| I\rangle i_{I}\right\rangle\left\langle i_{I}|\langle I|\bar{Y}| J\rangle| j_{J}\right\rangle\left\langle j_{J}|\langle J|\bar{Z}| K\rangle| k_{K}\right\rangle\left\langle k_{K}|\langle K|U| 0\rangle| 0_{0}\right\rangle}{\left(\omega_{i_{I}}+\omega_{x}\right)\left(\omega_{j_{J}}-\omega_{z}-\omega_{u}\right)\left(\omega_{k_{K}}-\omega_{u}\right)}\right. \\
& \left.-\sum_{\left(I, i_{I}\right),\left(J, j_{J}\right) \neq 0_{0}} \frac{\left.\left\langle 0_{0}|\langle 0|X| I\rangle| i_{I}\right\rangle\left\langle i_{I}|\langle I|Y| 0\rangle| 0_{0}\right\rangle\left\langle 0_{0}|\langle 0|Z| J\rangle| j_{J}\right\rangle\left\langle j_{J}|\langle J|U| 0\rangle| 0_{0}\right\rangle\right\rangle}{\left(\omega_{i_{I}}+\omega_{x}\right)\left(\omega_{j_{J}}+\omega_{z}\right)\left(\omega_{j_{J}}-\omega_{u}\right)}\right\}
\end{aligned}
$$

In square bracket notation the vibrational contributions to the mixed terms are:

$$
\langle\langle X, Y, Z, U\rangle\rangle_{\omega_{y}, \omega_{z}, \omega_{u}}^{\left[\alpha^{2}\right]}=P^{X Y Z U}\left\{\sum_{I, K \neq 0, j_{0} \neq 0_{0}} \frac{\left\langle 0_{0} \frac{\mid\langle 0|X| I\rangle\langle I|Y| 0\rangle}{\left(\omega_{I}+\omega_{x}\right)} j_{0}\right\rangle\left\langle j_{0} \mid \frac{\langle 0|Z| K\rangle\langle K|U| 0\rangle \mid}{\left(\omega_{K}-\omega_{u}\right)} 0_{0}\right\rangle}{\left(\omega_{j_{0}}-\omega_{z}-\omega_{u}\right)}\right\}
$$

as well as: 


$$
\begin{aligned}
\langle\langle X, Y, Z, U\rangle\rangle_{\omega_{y}, \omega_{2}, \omega_{u}}^{[\mu \beta]} & =P^{X Y Z U}\left\{\sum_{I, J \neq 0, k_{0} \neq 0_{0}} \frac{\left\langle 0_{0} \mid \frac{\langle 0|X| I\rangle\left\langle I\left|\bar{Y}^{e}\right| J\right\rangle\langle J|Z| 0\rangle \mid}{\left(\omega_{I}+\omega_{x}\right)\left(\omega_{J}-\omega_{z}-\omega_{u}\right) \mid} k_{0}\right\rangle\left\langle k_{0}|\langle 0|U| 0\rangle| 0_{0}\right\rangle}{\left(\omega_{k_{0}}-\omega_{u}\right)}\right. \\
& \left.+\sum_{i_{0} \neq 0_{0}, J, K \neq 0} \frac{\left\langle 0_{0}|\langle 0|X| 0\rangle| i_{0}\right\rangle\left\langle\left\langle i_{0}\left|\frac{\langle 0|Y| J\rangle\left\langle J\left|Z^{e}\right| K\right\rangle\langle K|U| 0\rangle \mid}{\left(\omega_{J}-\omega_{z}-\omega_{u}\right)\left(\omega_{K}-\omega_{u}\right) \mid}\right| 0_{0}\right\rangle\right.}{\left(\omega_{i_{0}}+\omega_{x}\right)}\right\}
\end{aligned}
$$

and 


$$
\begin{aligned}
& \langle\langle X, Y, Z, U\rangle\rangle_{\omega_{y}, \omega_{z}, \omega_{u}}^{\left[\mu^{2} \alpha\right]}=P^{X Y Z U} \\
& \times\left\{\sum_{I \neq 0, j_{0}, k_{0} \neq 0_{0}} \frac{\left\langle 0_{0}\left|\frac{\langle 0|X| I\rangle\langle I|Y| 0\rangle}{\left(\omega_{I}+\omega_{x}\right)}\right| j_{0}\right\rangle\left\langle j_{0}\left|\left\langle 0\left|\bar{Z}^{v}\right| 0\right\rangle\right| k_{0}\right\rangle\left\langle k_{0}|\langle 0|U| 0\rangle| 0_{0}\right\rangle}{\left(\omega_{j_{0}}-\omega_{z}-\omega_{u}\right)\left(\omega_{k_{0}}-\omega_{u}\right)}\right. \\
& +\sum_{J \neq 0, i_{0}, k_{0} \neq 0_{0}} \frac{\left\langle 0_{0}|\langle 0|X| 0\rangle| i_{0}\right\rangle\left\langle i_{0} \frac{\langle 0|Y| J\rangle\langle J|Z| 0\rangle}{\left(\omega_{J}-\omega_{z}-\omega_{u}\right)} \mid k_{0}\right\rangle\left\langle k_{0}|\langle 0|U| 0\rangle| 0_{0}\right\rangle}{\left(\omega_{i_{0}}+\omega_{x}\right)\left(\omega_{k_{0}}-\omega_{u}\right)} \\
& +\sum_{K \neq 0, i_{0}, j_{0} \neq 0_{0}} \frac{\left\langle 0_{0}|\langle 0|X| 0\rangle| i_{0}\right\rangle\left\langle i_{0}\left|\left\langle 0\left|\bar{Y}^{v}\right| 0\right\rangle\right| j_{0}\right\rangle\left\langle j_{0} \frac{|\langle 0|Z| K\rangle\langle K|U| 0\rangle|}{\left(\omega_{K}-\omega_{u}\right)} \mid 0_{0}\right\rangle}{\left(\omega_{i_{0}}+\omega_{x}\right)\left(\omega_{j_{0}}-\omega_{z}-\omega_{u}\right)} \\
& -\sum_{I \neq 0, j_{0} \neq 0_{0}} \frac{\left\langle 0_{0} \mid \frac{\langle 0|X| I\rangle\langle I|Y| 0\rangle \mid}{\left(\omega_{I}+\omega_{x}\right)} 0_{0}\right\rangle\left\langle 0_{0}|\langle 0|Z| 0\rangle| j_{0}\right\rangle\left\langle j_{0}|\langle 0|U| 0\rangle| 0_{0}\right\rangle}{\left(\omega_{j_{0}}+\omega_{z}\right)\left(\omega_{j_{0}}-\omega_{u}\right)} \\
& \left.-\sum_{J \neq 0, i_{0} \neq 0_{0}} \frac{\left\langle 0_{0} \mid\langle 0|X| 0\rangle i_{0}\right\rangle\left\langle i_{0}|\langle 0|Y| 0\rangle| 0_{0}\right\rangle\left\langle 0_{0}\left|\frac{\langle 0|Z| J\rangle\langle J|U| 0\rangle}{\left(\omega_{J}+\omega_{z}\right)\left(\omega_{J}-\omega_{u}\right) \mid}\right| 0_{0}\right\rangle}{\left(\omega_{i_{0}}+\omega_{x}\right)}\right\}
\end{aligned}
$$

An analysis similar to the one carried out in Ref. $^{8}$ may be done for the three square brackets in Eqs. (13)-(15). Since the derivations are a bit tedious we will not go through all the steps but will provide the reader with sufficient information to verify that the formulas given are correct. For $\left[\alpha^{2}\right]$ (Eq. (13)) and $\left[\mu^{2} \alpha\right]$ (Eq. (15)) no new material is required. The results are: 


$$
\begin{aligned}
& \left.\langle X, Y, Z, U\rangle\right|_{\omega_{y}, \omega_{z}, \omega_{u}} ^{\left[\alpha^{2}\right]}
\end{aligned}
$$

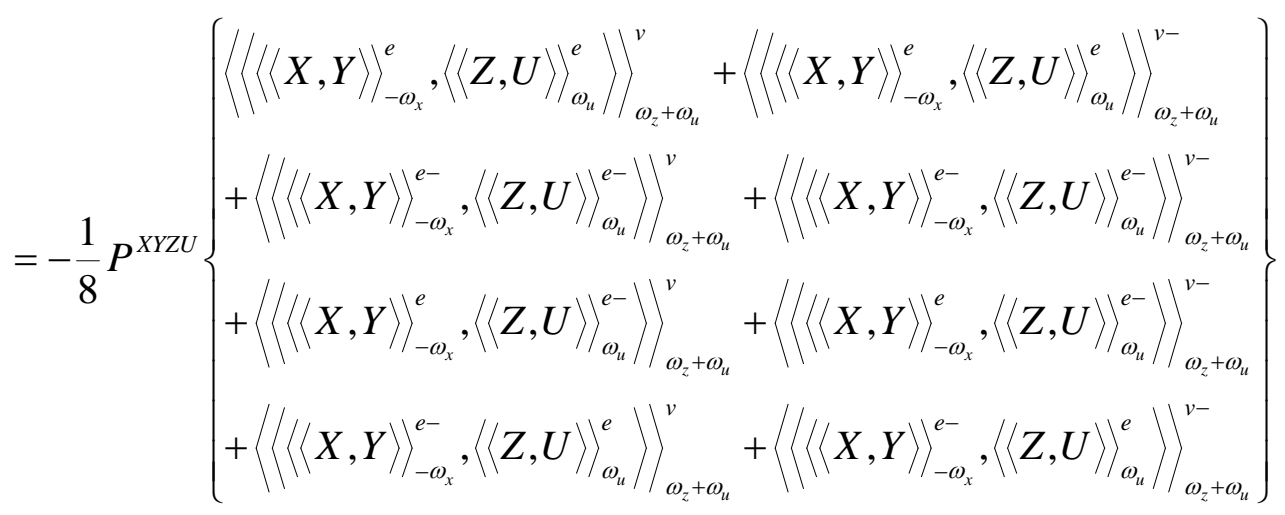

and

$$
\begin{aligned}
& \left.\langle X, Y, Z, U\rangle\right|_{\omega_{y}, \omega_{z}, \omega_{u}} ^{\left[\mu^{2} \alpha\right]}=\frac{1}{2} P^{X Y Z U} \\
& \times\left\{-\sum_{I \neq 0, j_{0}, k_{0} \neq 0_{0}} \frac{\left.\left.0_{0}(\langle Z, U\rangle)_{\omega_{u}}^{e}-\left.(Z, U)\right|_{\omega_{u}} ^{e-}\right) j_{0}\right)\left(j_{0}\left|\bar{Y}^{v}\right\rangle^{e}\left|k_{0}\right| k_{0}|X\rangle^{e}\left|0_{0}\right\rangle\right.}{\left(\omega_{j_{0}}+\omega_{z}+\omega_{u}\right)\left(\omega_{k_{0}}-\omega_{x}\right)}\right. \\
& -\sum_{K \neq 0, i_{0}, j_{0} \neq 0_{0}} \frac{\left.\left.\left\langle 0_{0} \mid X\right\rangle^{e}\left|i_{0}\right\rangle i_{0}\left|Y^{v}\right|^{e}\left|j_{0}\right\rangle j_{0}(\langle Z, U\rangle\rangle_{\omega_{u}}^{e}+\left.\langle Z, U\rangle\right|_{\omega_{u}} ^{e-}\right) 0_{0}\right\rangle}{\left(\omega_{i_{0}}+\omega_{x}\right)\left(\omega_{j_{0}}-\omega_{z}-\omega_{u}\right)} \\
& -\sum_{J \neq 0, i_{0}, k_{0} \neq 0_{0}} \frac{\left.\left\langle 0_{0} \mid X\right\rangle^{e}\left|i_{0}\right\rangle i_{0}\left|(\langle Z, U\rangle\rangle_{-\omega_{z}-\omega_{y}}^{e}-\langle Z, U\rangle\right\rangle_{-\omega_{z}-\omega_{y}}^{e-}\right) \mid k_{0}\left\langle k_{0}\left|Y^{e}\right| 0_{0}\right\rangle}{\left(\omega_{i_{0}}+\omega_{x}\right)\left(\omega_{k_{0}}-\omega_{y}\right)} \\
& \left.+\sum_{I \neq 0, j_{0} \neq 0_{0}} \frac{\left.\left.\left.\left.\left\langle 0_{0}\right|(\langle, U\rangle\rangle_{\omega_{u}}^{e}-(\langle, U\rangle)_{\omega_{u}}^{e-}\right) 0_{0}\right\rangle 0_{0}\left|Y^{e}\right| j_{0}\right\rangle j_{0}\left|\langle\rangle^{e}\right| 0_{0}\right\rangle}{\left(\omega_{j_{0}}+\omega_{y}\right)\left(\omega_{j_{0}}-\omega_{x}\right)}\right\}
\end{aligned}
$$

In Eq. (16) the terms on the first line involve only the symmetric electronic (quadratic) response function, whereas all the other terms contain the antisymmetric electronic (quadratic) response function at least once. The latter vanish in the static limit and, therefore, correspond to the missing terms. By the same argument the missing terms in Eq. (17) are: 


$$
\begin{aligned}
& \left\langle\left.\langle X, Y, Z, U\rangle\right|_{\omega_{y}, \omega_{z}, \omega_{u}} ^{\left[\mu^{2}\right]}=\frac{1}{2} P^{X Y Z U}\right. \\
& \times\left\{\sum_{I \neq 0, j_{0}, k_{0} \neq 0_{0}} \frac{\left.\left.\left.0_{0}|\langle Z, U)|_{\omega_{u}}^{e-}\left|j_{0} / j_{0}\right| Y^{v}\right|^{e} \mid k_{0}\right) k_{0}|X\rangle^{e} \mid 0_{0}\right)}{\left(\omega_{j_{0}}+\omega_{z}+\omega_{u}\right)\left(\omega_{k_{0}}-\omega_{x}\right)}\right. \\
& -\sum_{K \neq 0, i_{0}, j_{0} \neq 0_{0}} \frac{\left.\left.\left.0_{0}|X\rangle^{e}\left|i_{0} / i_{0}\right| Y^{v}\right\rangle^{e}\left|j_{0}\right\rangle j_{0} \mid\langle Z, U\rangle\right)_{\omega_{u}}^{e-} 0_{0}\right\rangle}{\left(\omega_{i_{0}}+\omega_{x}\right)\left(\omega_{j_{0}}-\omega_{z}-\omega_{u}\right)} \\
& +\sum_{J \neq 0, i_{0}, k_{0} \neq 0_{0}} \frac{\left.\left.0_{0}|X\rangle^{e}\left|i_{0}\right| i_{0} \mid\langle Z, U\rangle\right)_{-\omega_{z}-\omega_{y} \mid}^{e-} k_{0}\left|k_{0}\right| Y\right\rangle^{e}\left|0_{0}\right\rangle}{\left(\omega_{i_{0}}+\omega_{x}\right)\left(\omega_{k_{0}}-\omega_{y}\right)} \\
& \left.-\sum_{I \neq 0, j_{0} \neq 0_{0}} \frac{\left.\left.\left.0_{0} \mid\langle Z, U\rangle\right)_{\omega_{u}}^{e-} 0_{0}\right\rangle 0_{0}|Y\rangle^{e}\left|j_{0}\right\rangle j_{0}|X\rangle^{e} 0_{0}\right\rangle}{\left(\omega_{j_{0}}+\omega_{y}\right)\left(\omega_{j_{0}}-\omega_{x}\right)}\right\}
\end{aligned}
$$

For $[\mu \beta]$ (Eq. (14)) we need symmetrized cubic response functions. The totally symmetric cubic electronic response function is:

$$
\langle Y, Z, U\rangle\rangle_{\omega_{2}, \omega_{3}}^{e}=\frac{1}{\sqrt{6}} \sum_{J, K \neq 0}\left\{\begin{array}{l}
\frac{0|Y| J\rangle J|Z| K\rangle K|U| 0\rangle}{\left(\omega_{J}+\omega_{1}\right)\left(\omega_{K}-\omega_{3}\right)}+\frac{0|U| J\rangle J|Y| K\rangle K|Z| 0\rangle}{\left(\omega_{J}+\omega_{3}\right)\left(\omega_{K}-\omega_{2}\right)} \\
+\frac{0|Z| J\rangle J|U| K\rangle K|Y| 0\rangle}{\left(\omega_{J}+\omega_{2}\right)\left(\omega_{K}-\omega_{1}\right)}+\frac{0|Y| J\rangle J|U| K\rangle K|Z| 0\rangle}{\left(\omega_{J}+\omega_{1}\right)\left(\omega_{K}-\omega_{2}\right)} \\
+\frac{0|U| J|J| Z|K\rangle K|Y| 0\rangle}{\left(\omega_{J}+\omega_{3}\right)\left(\omega_{K}-\omega_{1}\right)}+\frac{0|Z| J\rangle J|\bar{Y}| K\rangle K|U| 0\rangle}{\left(\omega_{J}+\omega_{2}\right)\left(\omega_{K}-\omega_{3}\right)}
\end{array}\right\}
$$

There are 5 remaining symmetrized combinations. Of these, the two that will be used here are -

$$
\langle Y, Z, U\rangle\rangle_{\omega_{2}, \omega_{3}}^{e-1}=\frac{1}{\sqrt{6}} \sum_{J, K \neq 0}\left\{\begin{array}{l}
\frac{0|Y| J\rangle J|Z| K\rangle K|U| 0\rangle}{\left(\omega_{J}+\omega_{1}\right)\left(\omega_{K}-\omega_{3}\right)}+\frac{0|U| J\rangle J|\bar{Y}| K\rangle K|Z| 0\rangle}{\left(\omega_{J}+\omega_{3}\right)\left(\omega_{K}-\omega_{2}\right)} \\
+\frac{0|Z| J\rangle J|U| K\rangle K|Y| 0\rangle}{\left(\omega_{J}+\omega_{2}\right)\left(\omega_{K}-\omega_{1}\right)}-\frac{0|Y| J\rangle J|U| K\rangle K|Z| 0\rangle}{\left(\omega_{J}+\omega_{1}\right)\left(\omega_{K}-\omega_{2}\right)} \\
-\frac{0|U| J\rangle J|Z| K\rangle K|Y| 0\rangle}{\left(\omega_{J}+\omega_{3}\right)\left(\omega_{K}-\omega_{1}\right)}-\frac{0|Z| J\rangle J|\bar{Y}| K\rangle K|U| 0}{\left(\omega_{J}+\omega_{2}\right)\left(\omega_{K}-\omega_{3}\right)}
\end{array}\right\}
$$


and

$$
\langle Y, Z, U\rangle\rangle_{\omega_{2}, \omega_{3}}^{e-2}=\frac{1}{\sqrt{6}} \sum_{J, K \neq 0}\left\{\begin{array}{l}
2 \frac{0|Y| J\rangle J|Z| K\rangle K|U| 0\rangle}{\left(\omega_{J}+\omega_{1}\right)\left(\omega_{K}-\omega_{3}\right)}-\frac{0|U| J\rangle J|\bar{Y}| K\rangle K|Z| 0\rangle}{\left(\omega_{J}+\omega_{3}\right)\left(\omega_{K}-\omega_{2}\right)} \\
-\frac{0|Z| J\rangle J|\bar{U}| K\rangle K|Y| 0}{\left(\omega_{J}+\omega_{2}\right)\left(\omega_{K}-\omega_{1}\right)}
\end{array}\right\}
$$

In Eqs. (19)-(21) $\omega_{1}+\omega_{2}+\omega_{3}=0$. A combination of these equations yields:

$$
\left.\left.\left.\sum_{J, K \neq 0} \frac{0|Y| J\rangle J|Z| K\rangle K|U| 0\rangle}{\left(\omega_{J}+\omega_{1}\right)\left(\omega_{K}-\omega_{3}\right)}=\frac{1}{\sqrt{6}}\{\langle Y, Z, U\rangle\rangle_{\omega_{2}, \omega_{3}}+\langle Y, Z, U\rangle\right\rangle_{\omega_{2}, \omega_{3}}^{-1}+2\langle Y, Z, U\rangle\right\rangle_{\omega_{2}, \omega_{3}}^{-2}\right\}
$$

Finally, as a result of substituting this last relation into Eq. (14), we obtain:

$$
\begin{aligned}
& \left.\langle X, Y, Z, U\rangle\right|_{\omega_{y}, \omega_{z}, \omega_{u}} ^{[\mu \beta]}=\frac{1}{\sqrt{6}} P^{X Y Z U} \\
& \times\left\{\sum_{i_{0} \neq 0_{0}} \frac{\left.\left.\left.0_{0}\left|\{(Y, Z, U\rangle\rangle_{\omega_{z},-\omega_{y}-\omega_{z}}^{e}+\langle Y, Z, U\rangle\right|_{\omega_{z},-\omega_{y}-\omega_{z}}^{e-1}+2\langle Y, Z, U\rangle\right\rangle_{\omega_{z},-\omega_{y}-\omega_{z}}^{e-2}\right\} i_{0}\right\rangle i_{0}\left|\langle X\rangle_{0}^{e} 0_{0}\right\rangle}{\left(\omega_{i_{0}}-\omega_{x}\right)}\right. \\
& \left.+\sum_{i_{0} \neq 0_{0}} \frac{\left.\left.\left.\left.\left\langle 0_{0}\left|X^{e}\right| i_{0}\right\rangle i_{0} \mid\{(Y, Z, U\rangle)_{\omega_{2}, \omega_{u}}^{e}+\langle Y, Z, U\rangle\right)_{\omega_{z}, \omega_{u}}^{e-1}+2\langle Y, Z, U\rangle\right)_{\omega_{z}, \omega_{u}}^{e-2}\right\}_{0}\right\rangle}{\left(\omega_{i_{0}}+\omega_{x}\right)}\right\}
\end{aligned}
$$

Again, the missing terms are those that contain electronic response functions other than the totally symmetric one. In this case we have: 


$$
\begin{aligned}
& \frac{1}{\sqrt{6}} P^{X Y Z U}\left\{\sum_{i_{0} \neq 0_{0}} \frac{\left\langle 0_{0}\left|\langle\langle Y, Z, U\rangle\rangle_{\omega_{2},-\omega_{y}-\omega_{z} \mid}^{e-1}\right| i_{0}\right\rangle\left\langle i_{0}\left|\langle X\rangle^{e}\right| 0_{0}\right\rangle}{\left(\omega_{i_{0}}-\omega_{x}\right)}\right. \\
& +2 \sum_{i_{0} \neq 0_{0}} \frac{\left\langle 0_{0}\left|\langle\langle Y, Z, U\rangle\rangle_{\omega_{2},-\omega_{y}-\omega_{z} \mid}^{e-2}\right| i_{0}\right\rangle\left\langle i_{0}\left|\langle X\rangle^{e}\right| 0_{0}\right\rangle}{\left(\omega_{i_{0}}-\omega_{x}\right)}+\sum_{i_{0} \neq 0_{0}} \frac{\left\langle 0_{0}\left|\langle X\rangle^{e}\right| i_{0}\right\rangle\left\langle i_{0}\left|\langle\langle Y, Z, U\rangle\rangle_{\omega_{2}, \omega_{u}}^{e-1}\right| 0_{0}\right\rangle}{\left(\omega_{i_{0}}+\omega_{x}\right)} \\
& \left.+2 \sum_{i_{0} \neq 0_{0}} \frac{\left.\left\langle 0_{0}\left|\langle X\rangle^{e}\right| i_{0}\right\rangle\left\langle i_{0}\left|\langle\langle Y, Z, U\rangle\rangle_{\omega_{2}, \omega_{u}}^{e-2}\right| 0_{0}\right\rangle\right\}}{\left(\omega_{i_{0}}+\omega_{x}\right)}\right\}
\end{aligned}
$$

\section{Contribution of missing terms to NLO processes in IOF approximation.}

As discussed in Sec. II the missing term for the dynamic vibrational first hyperpolarizability is:

$$
\frac{1}{2} P^{X Y Z}\left\langle\left\langle\langle X\rangle^{e},\langle\langle Y, Z\rangle\rangle_{\omega_{z}}^{e-}\right\rangle\right\rangle_{-\omega_{x}}^{v-}
$$

The dc-Pockels effect corresponds to the case where the optical frequencies are $\omega_{x}=-\omega, \omega_{y}=\omega, \omega_{z}$ $=0$. In Eq. (25) the operator $P^{X Y Z}$ generates six permutations. From Eq. (10) we know that four of the six are zero, two because of the electronic asymmetric quadratic response formula and two because of the asymmetric vibrational quadratic response expression. Thus, the only two permutations that survive are:

$$
\left.\left.\left.\left.\langle X\rangle^{e},\langle Z, Y\rangle\right\rangle_{\omega}^{e-}\right\rangle_{\omega}^{v-} \text { and }\left\langle\langle Y\rangle^{e},\langle Z, X\rangle\right\rangle_{-\omega}^{e-}\right\rangle\right\rangle_{-\omega}^{v-}
$$

In the IOF approximation for the vibrational quadratic response we set $\omega=\infty$ and, in that event, it follows from the definition of Eq. (5) that both permutations in (26) are zero. Hence, the missing term vanishes for the dc-Pockels effect. This is the only second order dynamic NLO 
process for which there exists a vibrational contribution in the IOF approximation. There is no contribution, for example, to second harmonic generation. On the other hand, in third order, there are vibrational contributions to several dynamic NLO processes including the optical Kerr effect (OKE), electric field induced second harmonic generation (EFISH) and the intensity-dependent refractive index (IDRI), also known as degenerate four-wave mixing (DFWM). The various square brackets may contribute to one or more of these processes. ${ }^{26,33}$

\section{1. $\quad\left[\alpha^{2}\right]$}

The $\left[\alpha^{2}\right]$ square bracket (see Eq. (16)) contributes to the OKE as well as IDRI. As indicated earlier the missing terms in Eq. (16) are:

$$
-\frac{1}{8} P^{X Y Z U}\left\{\begin{array}{l}
\left.\left.\left.\left\langle\langle\langle, Y\rangle\rangle_{-\omega_{x}}^{e-},\langle Z, U\rangle\right)_{\omega_{u}}^{e-}\right\rangle_{\omega_{z}+\omega_{u}}^{v}+\left\langle\langle\langle X, Y\rangle\rangle_{-\omega_{x}}^{e-},\langle Z, U\rangle\right\rangle_{\omega_{u}}^{e-}\right\rangle\right\rangle_{\omega_{z}+\omega_{u}}^{v-} \\
\left.\left.+\left\langle\langle\langle X, Y\rangle\rangle_{-\omega_{x}}^{e},\left.\langle Z, U\rangle\right|_{\omega_{u}} ^{e-}\right\rangle_{\omega_{z}+\omega_{u}}^{v}+\left\langle\langle\langle X, Y\rangle\rangle_{-\omega_{x}}^{e},\langle Z, U\rangle\right\rangle_{\omega_{u}}^{e-}\right\rangle\right\rangle_{\omega_{z}+\omega_{u}}^{v-} \\
\left.\left.+\left\langle\langle\langle X, Y\rangle\rangle_{-\omega_{x}}^{e-},\left.\langle Z, U\rangle\right|_{\omega_{u}} ^{e}\right\rangle_{\omega_{z}+\omega_{u}}^{v}+\left\langle\langle\langle X, Y\rangle\rangle_{-\omega_{x}}^{e-},\langle Z, U\rangle\right\rangle_{\omega_{u}}^{e}\right\rangle\right\rangle_{\omega_{z}+\omega_{u}}^{v-}
\end{array}\right\}
$$

For the OKE we consider the set of frequencies $\omega_{x}=-\omega, \omega_{y}=\omega, \omega_{z}=0, \omega_{u}=0$. There are 24 permutations of the operator/frequency pairs, generated by $P^{X Y Z U}$, that we need to consider. In the second term on the rhs of Eq. (27) 20 of the 24 permutations give a zero contribution by virtue of Eq. (10). If we apply the IOF approximation, the remaining 4 permutations, such as:

$$
\left.\left.\left.\langle Y, X\rangle\rangle_{-\omega}^{e-},\langle Z, U\rangle\right\rangle_{\omega}^{e-}\right\rangle\right\rangle_{\infty}^{v-}=0
$$

are zero. For the same reasons the first, fourth, and sixth terms of Eq. (27) are zero. However, the third and fifth terms each have four permutations that survive yielding: 


$$
\begin{aligned}
& \left.\left.\left.\left.\left.\langle U, Z\rangle_{0}^{e},\langle Y, X\rangle\right\rangle_{-\omega}^{e-}\right\rangle\right\rangle_{0}^{v}+\left\langle\langle\langle Z, U\rangle)_{0}^{e},\langle Y, X\rangle\right\rangle_{-\omega}^{e-}\right\rangle\right\rangle_{0}^{v} \\
& \left.\left.\left.\left.+\langle\langle U, Z\rangle)_{0}^{e},\langle X, Y\rangle\right\rangle_{\omega}^{e-}\right\rangle\right\rangle_{0}^{v}+\left\langle\langle\langle Z, U\rangle)_{0}^{e},\langle\langle X, Y\rangle\rangle_{\omega}^{e-}\right\rangle\right\rangle_{0}^{v} \\
& \left.\left.\left.\left.-\left\langle\langle\langle Y, X\rangle\rangle_{-\omega}^{e-},\langle Z, U\rangle\right)_{0}^{e}\right\rangle\right\rangle_{0}^{v}-\left\langle\langle\langle Y, X\rangle)_{-\omega}^{e-},\langle U, Z\rangle\right\rangle_{0}^{e}\right\rangle\right\rangle_{0}^{v} \\
& \left.\left.\left.\left.-\left\langle\langle\langle X, Y\rangle\rangle_{\omega}^{e-},\langle Z, U\rangle\right)_{0}^{e}\right\rangle\right\rangle_{0}^{v}-\left\langle\langle\langle X, Y\rangle\rangle_{\omega}^{e-},\langle U, Z\rangle\right\rangle_{0}^{e}\right\rangle\right\rangle_{0}^{v}
\end{aligned}
$$

In Eq. (29) half the terms contain the factor $\left\langle\langle Y, X\rangle_{-\omega}^{e^{-}}\right.$. These can be converted to $\left\langle\langle X, Y\rangle_{\omega}^{e^{-}}\right.$using Eqs. (8) and (9). After that is done, it is readily seen that the terms 1 and 3, 2 and 4, etc. all cancel in pairs. Thus, once again the contribution of the missing terms is zero in the IOF approximation.

For the IDRI we set $\omega_{x}=-\omega, \omega_{y}=\omega, \omega_{z}=-\omega, \omega_{u}=\omega$. In that event the second, fourth, and sixth terms in Eq. (27) are zero either because of Eq. (10), as applied to the sum over vibrational levels, or the IOF approximation. For the first, third, and fifth terms the IOF approximation removes 8 of the 24 permutations. Then, taking advantage of the relation:

$$
P^{Z U}\langle\langle, U\rangle\rangle_{\omega_{2}}^{e^{-}}=0
$$

it is easy to show that the sum of the sixteen surviving terms is zero.

\section{2. $\left[\mu^{2} \alpha\right]$}

The missing $\left[\mu^{2} \alpha\right]$ terms are given by Eq. (18). Of the three major third order NLO processes this square bracket contributes only to the OKE. Using the frequencies given immediately below Eq. (27), as well as Eq. (10) and the IOF approximation, we find that only 4 of the 24 permutations survive for each of the missing terms. It is, then, easy to verify that these 4 permutations cancel one another. 


\section{3. $[\mu \beta]$}

Finally, we come to $[\mu \beta]$, which contributes to both the OKE and EFISH. The missing terms are given by Eq. (24). As far as OKE is concerned, we make use of Eqs. (20) and (21) which lead to:

$$
\langle\langle Y, Z, U\rangle\rangle_{\omega_{2}, \omega_{3}}^{-1}+\langle\langle Z, Y, U\rangle\rangle_{\omega_{1}, \omega_{3}}^{-1}=0
$$

and

$$
\langle\langle Y, Z, U\rangle\rangle_{\omega_{2}, \omega_{3}}^{-2}+\langle\langle U, Y, Z\rangle\rangle_{\omega_{1}, \omega_{2}}^{-2}+\langle\langle Z, U, Y\rangle\rangle_{\omega_{3}, \omega_{1}}^{-2}=0
$$

Upon application of the permutation operator the last two relations yield:

$$
P^{Y Z U}\langle\langle Y, Z, U\rangle\rangle_{\omega_{2}, \omega_{3}}^{-1}=0
$$

and

$$
P^{Y Z U}\langle\langle Y, Z, U\rangle\rangle_{\omega_{2}, \omega_{3}}^{-2}=0
$$

Of the 24 permutations in Eq. (24) half are zero due to the IOF approximation. For the remaining permutations of the first and second term $\omega_{u}=-\omega_{y}-\omega_{z}$. It, then, follows from Eqs. (33) and (34) that these permutations vanish, as do all the remaining permutations of the third and fourth terms.

For EFISH $\omega_{x}=-2 \omega, \omega_{y}=\omega, \omega_{z}=\omega, \omega_{u}=0$. After applying the IOF approximation only six permutations of each term remain. For instance, the first term of Eq. (24) reduces to: 


$$
\begin{aligned}
& \sum_{i_{0} \neq 0_{0}}\left\{\frac{\left\langle 0_{0} \mid\langle X, Y, Z\rangle\right\rangle_{\omega, \omega}^{e-1} \mid i_{0}\left\langle i_{0}\left|U^{e}\right| 0_{0}\right\rangle}{\omega_{i_{0}}}+\frac{\left.\left.\left\langle 0_{0}\right|\langle Y, Z, X\rangle\right)_{\omega,-2 \omega}^{e-1}\left|i_{0}\right\rangle / i_{0}\left|U^{e}\right| 0_{0}\right\rangle}{\omega_{i_{0}}}\right. \\
& \frac{\left\langle 0_{0} \mid\langle X, Z, Y\rangle\right\rangle_{\omega, \omega}^{e-1}\left|i_{0}\right\rangle i_{0}|U\rangle^{e}\left|0_{0}\right\rangle}{\omega_{i_{0}}}+\frac{\left.\left\langle 0_{0} \mid\langle Y, X, Z\rangle\right\rangle_{-2 \omega, \omega}^{e-1}\left|i_{0}\right\rangle i_{0}\left|U^{e}\right| 0_{0}\right\rangle}{\omega_{i_{0}}} \\
& \left.\frac{\left.\left\langle 0_{0} \mid\langle Z, X, Y\rangle\right\rangle_{-2 \omega, \omega}^{e-1}\left|i_{0}\right\rangle i_{0}\left|U^{e}\right| 0_{0}\right\rangle}{\omega_{i_{0}}}+\frac{\left.\left.0_{0} \mid\langle Z, Y, X\rangle\right)_{\omega,-2 \omega}^{e-1}\left|i_{0}\right\rangle i_{0}\left|U^{e}\right| 0_{0}\right\rangle}{\omega_{i_{0}}}\right\}=0
\end{aligned}
$$

This sum vanishes by virtue of Eq. (33). The same is true of the third term, whereas the second and fourth terms are zero due to Eq.(34).

\section{Conclusions and perspective.}

In the response theory treatment of second order vibrational NLO properties, as formulated by Hansen et al., ${ }^{8}$ a term appears that is missing in the original BK perturbation method due to an explicit approximation made in the latter. This so-called missing term vanishes exactly in the static limit. It is shown here that it also vanishes in the infinite optical frequency approximation. Moreover, we have extended the response theory treatment to third order properties and have reached the same general conclusion in that case as well. Our results appear to justify the approximation made in the original BK method. Of course, the term in question may still be important at finite non-zero frequencies, especially on a relative basis, and should certainly be taken into account when high accuracy is desired. The initial calculations carried out by Hansen, et al. are consistent with this interpretation.

\section{ACKNOWLEDGMENTS}

Financial help has been furnished by the Spanish MEC Project No. CTQ2008-06696/BQU 
and by the catalan Departament d’Universitats, Recerca i Societat de la Informació (DURSI) through project No. $2009 S G R 637$.

\section{References}

1. Bishop, D. M.; Norman, P. In Handbook of Advanced Electronic and Photonic Materials and Devices; Academic Press: San Diego, 2001.

2. $\quad$ Bishop, D. M. In Advances in Chemical Physics, Vol 104, 1998, p 1-40.

3. Bishop, D. M.; Kirtman, B. Journal of Chemical Physics 1991, 95, 2646-2658.

4. $\quad$ Bishop, D. M.; Kirtman, B. J Chem Phys 1992, 97, 5255-5256.

5. $\quad$ Bishop, D. M.; Luis, J. M.; Kirtman, B. J Chem Phys 1998, 108, 10013-10017.

6. $\quad$ Bishop, D. M.; Dalskov, E. K. J Chem Phys 1996, 104, 1004-1011.

7. Thorvaldsen, A. J.; Ruud, K.; Jaszunski, M. J Phys Chem A 2008, 112, 11942-11950.

8. Hansen, M. B.; Christiansen, O.; Hattig, C. J Chem Phys 2009, 131, 154101.

9. $\quad$ Luis, J. M.; Champagne, B.; Kirtman, B. Int J Quantum Chem 2000, 80, 471-479.

10. Bishop, D. M.; Kirtman, B.; Kurtz, H. A.; Rice, J. E. J Chem Phys 1993, 98, 8024-8030.

11. Bishop, D. M.; Sauer, S. P. A. J Chem Phys 1997, 107, 8502-8509.

12. Kirtman, B.; Toto, J. L.; Breneman, C.; de Melo, C. P.; Bishop, D. M. J Chem Phys 1998, 108, 4355-4357.

13. Pessoa, R.; Castro, M. A.; Amaral, O. A. V.; Fonseca, T. L. Chem Phys Lett 2005, 412, 16-22.

14. Marti, J.; Bishop, D. M. J Chem Phys 1993, 99, 3860-3864.

15. Luis, J. M.; Duran, M.; Champagne, B.; Kirtman, B. J Chem Phys 2000, 113, 5203-5213.

16. Bishop, D. M.; Kirtman, B.; Champagne, B. J Chem Phys 1997, 107, 5780-5787.

17. Bishop, D. M.; Pipin, J.; Kirtman, B. J Chem Phys 1995, 102, 6778-6786.

18. Bishop, D. M.; Norman, P. J Chem Phys 1999, 111, 3042-3050.

19. Luis, J. M.; Duran, M.; Kirtman, B. J Chem Phys 2001, 115, 4473-4483.

20. Luis, J. M.; Duran, M.; Andres, J. L.; Champagne, B.; Kirtman, B. J Chem Phys 1999, $111,875-884$.

21. Bishop, D. M.; Kirtman, B. J Chem Phys 1998, 109, 9674-9676.

22. Dutra, A. S.; Castro, M. A.; Fonseca, T. L.; Fileti, E. E.; Canuto, S. J Chem Phys 2010, 132, 034307.

23. Avramopoulos, A.; Jablonski, M.; Papadopoulos, M. G.; Sadlej, A. J. Chem Phys 2006, 328, 33-44.

24. Christiansen, O.; Kongsted, J.; Paterson, M. J.; Luis, J. M. J Chem Phys 2006, 125, 214309.

25. Bishop, D. M.; Kirtman, B. Phys Rev B 1997, 56, 2273-2274.

26. Luis, J. M.; Marti, J.; Duran, M.; Andres, J. L.; Kirtman, B. J Chem Phys 1998, 108, 4123-4130.

27. Zalesny, R.; Papadopoulos, M. G.; Bartkowiak, W.; Kaczmarek, A. J Chem Phys 2008, $129,134310$.

28. Tomasi, J.; Mennucci, B.; Cammi, R. Chem Rev 2005, 105, 2999-3093.

29. Bishop, D. M.; Champagne, B.; Kirtman, B. J Chem Phys 1998, 109, 9987-9994.

30. Jacquemin, D.; Champagne, B.; Perpete, E. A.; Luis, J. M.; Kirtman, B. J Phys Chem A 2001, 105, 9748-9755. 
31. Kirtman, B.; Luis, J. M.; Bishop, D. M. J Chem Phys 1998, 108, 10008-10012.

32. Kirtman, B.; Luis, J. M. J Chem Phys 2008, 128, 114101.

33. Bishop, D. M.; Hasan, M.; Kirtman, B. J Chem Phys 1995, 103, 4157-4159.

34. Squitieri, E.; Landaeta, C. M. Mol Phys 2008, 106, 1341-1352.

35. $\quad$ Luis, J. M.; Duran, M.; Andres, J. L. J Chem Phys 1997, 107, 1501-1512.

36. Bishop, D. M.; Pipin, J. J Chem Phys 1995, 103, 4980-4984.

37. Luis, J. M.; Reis, H.; Papadopoulos, M.; Kirtman, B. J Chem Phys 2009, 131, 034116.

38. Luis, J. M.; Torrent-Sucarrat, M.; Christiansen, O.; Kirtman, B. J Chem Phys 2007, 127, 084118.

39. Torrent-Sucarrat, M.; Luis, J. M.; Kirtman, B. J Chem Phys 2005, 122, 204108.

40. $\quad$ Bishop, D. M.; Luis, J. M.; Kirtman, B. J Chem Phys 2002, 116, 9729-9739.

41. Cohen, M. J.; Willetts, A.; Amos, R. D.; Handy, N. C. J Chem Phys 1994, 100, 4467-

4476.

42. $\quad$ Norman, P.; Luo, Y.; Agren, H. J Chem Phys 1998, 109, 3580-3588.

43. Eckart, U.; Ingamells, V. E.; Papadopoulos, M. G.; Sadlej, A. J. J Chem Phys 2001, 114, 735-745.

44. Santiago, E.; Castro, M. A.; Fonseca, T. L.; Mukherjee, P. K. J Chem Phys 2008, 128, 064310.

45. Perpete, E. A.; Quinet, O.; Champagne, B. J Opt A: Appl Opt 2000, 2, 247-254.

46. Bishop, D. M.; Bouferguene, A. Int J Quantum Chem 2000, 78, 348-377.

47. Quinet, O.; Champagne, B.; Kirtman, B. J Mol Struct (Theochem) 2003, 633, 199-207.

48. Loboda, O.; Zalesny, R.; Avramopoulos, A.; Luis, J. M.; Kirtman, B.; Tagmatarchis, N.;

Reis, H.; Papadopoulos, M. G. J Phys Chem A 2009, 113, 1159-1170.

49. Torrent-Sucarrat, M.; Sola, M.; Duran, M.; Luis, J. M.; Kirtman, B. J Chem Phys 2004, 120, 6346-6355.

50. Torrent-Sucarrat, M.; Sola, M.; Duran, M.; Luis, J. M.; Kirtman, B. J Chem Phys 2003, 118, 711-718.

51. Prasad, P. N.; Williams, D. J. Introduction to Nonlinear Optical Effects in Molecules and Polymers; Wiley: New York, 1990.

52. Asselberghs, I.; Hennrich, G.; McCleverty, J.; Boubekeur-Lecaque, L.; Coe, B. J.; Clays, K. In ORGANIC OPTOELECTRONICS AND PHOTONICS III; Heremans, P. L.; Muccini, M.; Meulenkamp, E. A., Eds.; SPIE-INT SOC OPTICAL ENGINEERING: Strasbourg, 2008, p p. W9991.

53. Torrent-Sucarrat, M.; Sola, M.; Duran, M.; Luis, J. M.; Kirtman, B. J Chem Phys 2002, 116, 5363-5373.

54. Quinet, O.; Champagne, B. J Chem Phys 1998, 109, 10594-10602.

55. Christiansen, O. J Chem Phys 2005, 122, 194105. 